\title{
Upward Drawings on Planes and Spheres
} Extended Abstract for Graph Drawing '95

20 - 22 September 1995

\author{
S. Mehdi Hashemi ${ }^{1}$, Andrzej Kisielewicz ${ }^{2}$ and Ivan Rival ${ }^{3}$ \\ 1 Department of Mathematics \\ University of Ottawa \\ Ottawa K1N 6N5 Canada \\ shashemiocsi.uottara.ca \\ 2 Mathematical Institute \\ University of Wroclaw \\ Wroclaw, Poland \\ kisielomath. uni.wroc.pl \\ 3 Department of Computer Science \\ University of Ottawa \\ Ottara K1N 6N5 Canada \\ rivalocsi.uottana.ca
}

\begin{abstract}
Although there is a linear time algorithm to decide whether an ordered set has an upward drawing on a surface topologically equivalent to a sphere, we shall prove that the decision problem whether an ordered set has an upward drawing on a sphere itself is NP-complete. To this end we explore the surface topology of ordered sets highlighting especially the role of their saddle points.
\end{abstract}

\section{Introduction}

The search for an efficient upward planarity testing algorithm for ordered sets ${ }^{4}$ is a longstanding problem, much sought after by theoreticians of graphical data structures. It has always been a mystery how upward planarity testing for orders could be so difficult if its undirected companion, planarity-testing for graphs is so easy. In spite of well-known linear time algorithms for graph planarity testing (e.g. [Hopcroft and Tarjan 1974]), it is not even self-evident that there is a finite algorithm for upward planarity testing.

For many years progress has been slow.

- Planar lattices are dismantlable [Baker, Fishburn and Roberts 1971]

- Algorithmics of planar lattices [Kelly and Rival 1975].

- Planarity-testing for lattices using graph planarity-testing [Platt 1976].

- Straight lines for planar upward drawings [Kelly 1987] .

- Bipartite planar upward drawings [di Battista, Liu and Rival 1990].

${ }^{4}$ In fact, by adjoining subdivision points as needed we may just as well consider directed acyclic graphs. 


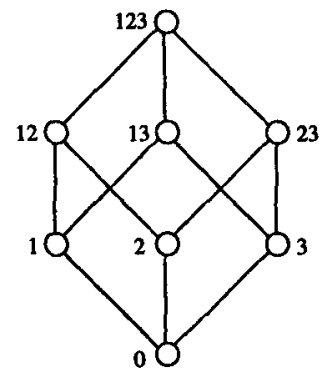

Three-dimensional hypercube

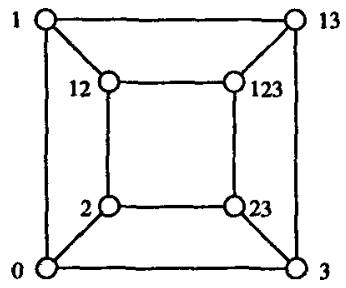

Its covering graph

Fig. 1. The three-dimensional hypercube is a nonplanar ordered set (with planar covering graph).

- Planar ordered sets of width two [Czyzowicz, Pelc and Rival 1990].

- Planar ordered sets with bottom [Hutton and Lubiw 1991].

- Planar triangle-free graphs have planar orientations [Kisielewicz and Rival 1993].

Recently, [A. Garg and R. Tamassia (1995)] made a major breakthrough. By transforming the NOT-ALL-EQUAL-3-SAT decision problem into an auxiliary flow decision problem with integer coordinates, and then providing yet another transformation to upward planarity, they proved that upward planaritytesting is NP-complete.

The results presented here are based on our work of recent years whose twofold purpose is to understand upward drawings of orders through the twodimensional surfaces in $\mathbf{R}^{\mathbf{3}}$ on which they may be embedded (without crossing edges), and conversely, to better understand two-dimensional surfaces in $\mathbf{R}^{3}$ by means of the upward drawings that fit on them (cf. [Hashemi and Rival (1994)].

Our main result bears on this problem.

\section{Upward Sphericity Testing}

INSTANCE Given an ordered set $P$.

QUESTION Does $P$ have an upward drawing on the sphere $\left\{(x, y, z): x^{2}+y^{2}+\right.$ $\left.z^{2}=1\right\}$ without the crossing of edges?

Although much is already written about planarity for graphs and ordered sets, there is much less about sphericity. Indeed, apart from bits and pieces in the topological graph theory and differential geometry literature there is virtually a blank about the algorithmics of sphericity. 


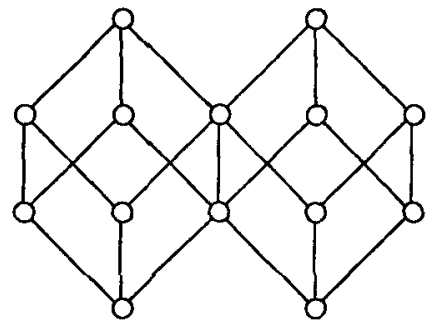

The doublecube

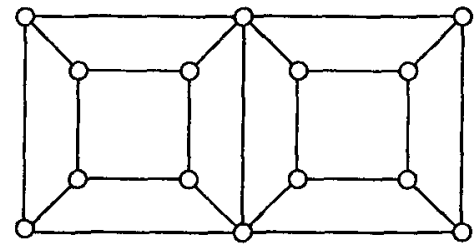

Its planar covering graph

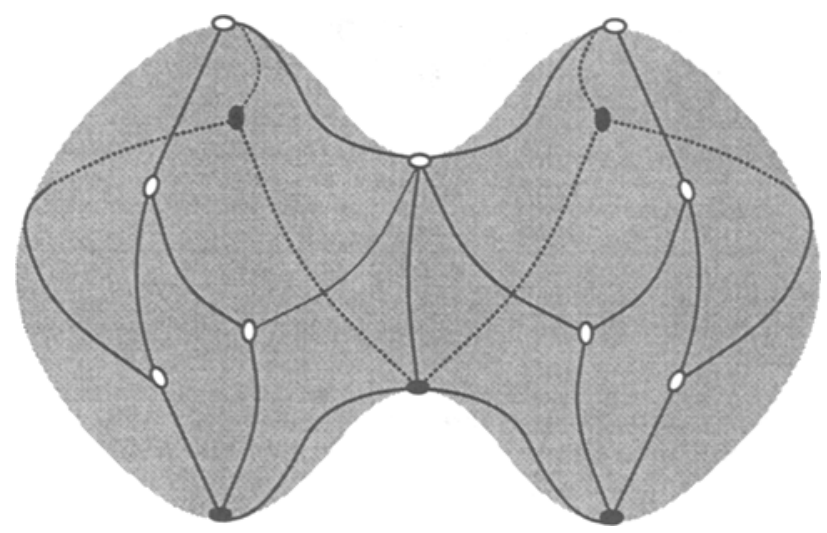

Upward drawing with saddle points

Fig. 2. Upward drawing of the doublecube on a surface of genus zero.

Like upward planarity testing the complexity of upward sphericity testing seems far from obvious. Indeed, according to [Ewacha, Li and Rival (1991)] - and unlike upward planarity testing, the decision problem whether an ordered set has an upward drawing on a surface of genus zero (that is, a topological sphere) is itself polynomial. The reason is that its planar covering graph can be "lifted" to an upward drawing on a surface of genus zero. On the other hand, it is our main result that upward sphericity is still NP-complete.

The two-dimensional cube $\{\mathbf{0 , 1}, \mathbf{2}, \mathbf{1 2}\}$ has an upward drawing on the plane, (e.g. $\mathbf{0}=(1,0,0), \mathbf{1}=(0,1,0), \mathbf{2}=(2,1,0), \mathbf{1 2}=(1,2,0))$ : the two-dimensional cube is a planar ordered set. The three-dimensional cube (cf. Figure 1 ) is a nonplanar ordered set.

The three-dimensional cube, although nonplanar, has an upward drawing 


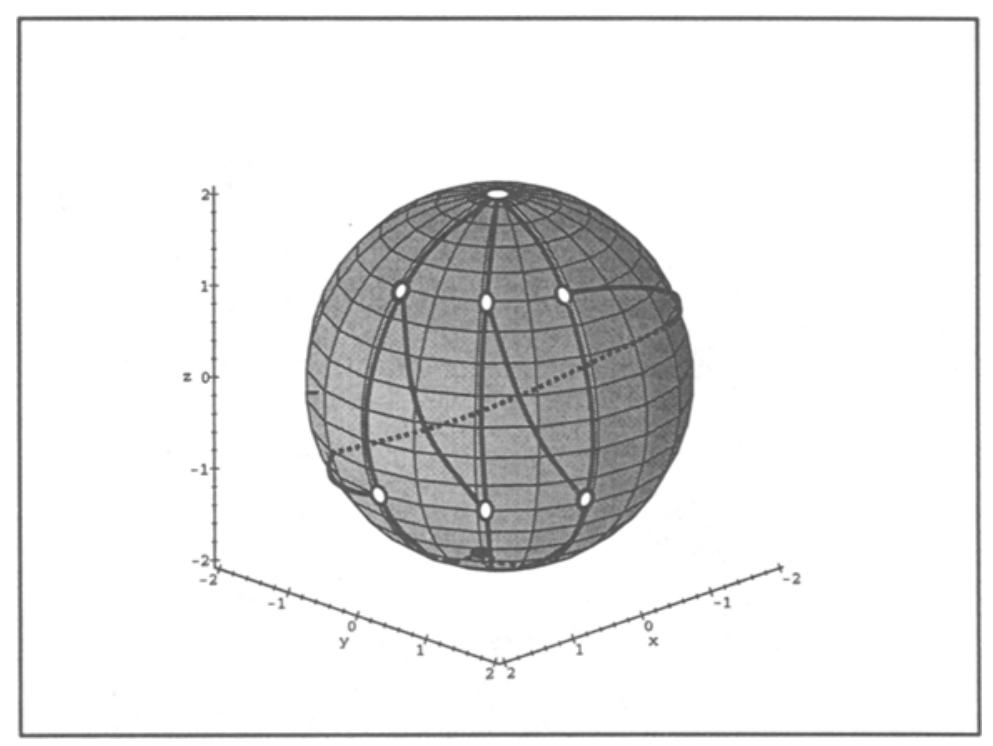

Fig. 3. Upward drawing of the three-dimensional hypercube on the sphere.

on the sphere, (e.g. with vertex placement according to $\mathbf{0}=(0,0,-1), \mathbf{1}=$ $\left(\frac{1}{\sqrt{2}}, 0,-\frac{1}{\sqrt{2}}\right), 2=\left(0, \frac{1}{\sqrt{2}},-\frac{1}{\sqrt{2}}\right), 3=\left(-\frac{1}{\sqrt{2}}, 0,-\frac{1}{\sqrt{2}}\right), 12=\left(\frac{1}{\sqrt{2}}, 0, \frac{1}{\sqrt{2}}\right), 23=$ $\left(0, \frac{1}{\sqrt{2}}, \frac{1}{\sqrt{2}}\right), \mathbf{1 3}=\left(-\frac{1}{\sqrt{2}}, 0, \frac{1}{\sqrt{2}}\right), \mathbf{1 2 3}=(0,0,1)$, and monotonic arcs joining the appropriate pairs of vertices - cf. Figure 1 and Figure 3 ). The doublecube, on the other hand, while it has a planar covering graph, and has an upward drawing on a surface with no handles or holes, has no upward drawing on a sphere: any smooth surface in $\mathbf{R}^{3}$ on which the doublecube has an upward drawing has a saddle point (cf. Figure 2).

Theorem 1. Upward sphericity testing is NP-complete.

The proof, in part, runs parallel to this important recent result of [A. Garg and R. Tamassia (1995)]. Despite initial appearances, though, we see no way to derive our theorem, directly, or by way of corollary, from it.

Theorem 2. Upward planarity testing is NP-complete.

In the process, we shall derive a proof (which seems to us simpler and more transparent) based on

Exact Cover By 3-Sets 
INSTANCE Given a set $X$ with $|X|=3 q$ and a collection $C$ of 3-element subsets of $X$.

QUESTION Does $C$ contain an exact cover for $X$, i.e., a subcollection $C^{\prime} \subseteq C$ such that every element of $X$ occurs in exactly one member of $C^{\prime}$ ?

Both the present proof, and the earlier one of [A. Garg and R. Tamassia (1995)], rely on "gadgets" that they call tendrils and wiggles, in our jargon spirals. An example, $S(1,0)$ a spiral with a "frame" illustrated in Figure 4, has a planar covering graph and yet no upward drawing on a plane. Moreover, although it does have an upward drawing on a smooth surface of genus one, such a surface must always have a saddle point. The argument is based on this technical result which seems to be of independent interest.

Theorem 3. Let $P$ be an ordered set, let $S$ be a smooth surface in $\mathbf{R}^{3}$ on which $P$ has an upward drawing. Let $F$ be a face of this upward drawing and let $m$ stand for the number of paths of vertices of this face $F$, all members of which are interior, or all members of which are extremal. If

$$
\text { interior }(F)>\operatorname{extremal}(F)+2 m
$$

then $S$ must have a saddle point.

Although upward planarity testing is difficult, it may be that, if it is known that an ordered set already has an upward drawing on a genus zero surface then there is an efficient procedure to decide whether it has an upward drawing on a plane, too.

\section{Upward Planarity Testing of Spherical Ordered Sets}

INSTANCE Given an ordered set $P$ with upward drawing on a sphere. QUESTION Does $P$ have an upward drawing on a plane?

Problem 4. Is Upward Planarity Testing of Spherical Ordered Sets polynomial?

Our analysis here has made no distinction between one or more saddle points.

\section{$m$-Saddle Point Surfaces}

INSTANCE Given an ordered set $P$ with planar covering graph and a nonnegative integer $s$.

QUESTION Does $P$ have an upward drawing on a smooth surface of genus zero with at most $s$ saddle points?

Problem 5. Is the $s$-saddle point problem NP-complete?

In spite of the intractability of upward spherical testing it is still of interest to know just which ordered sets have an upward drawing on a sphere. 


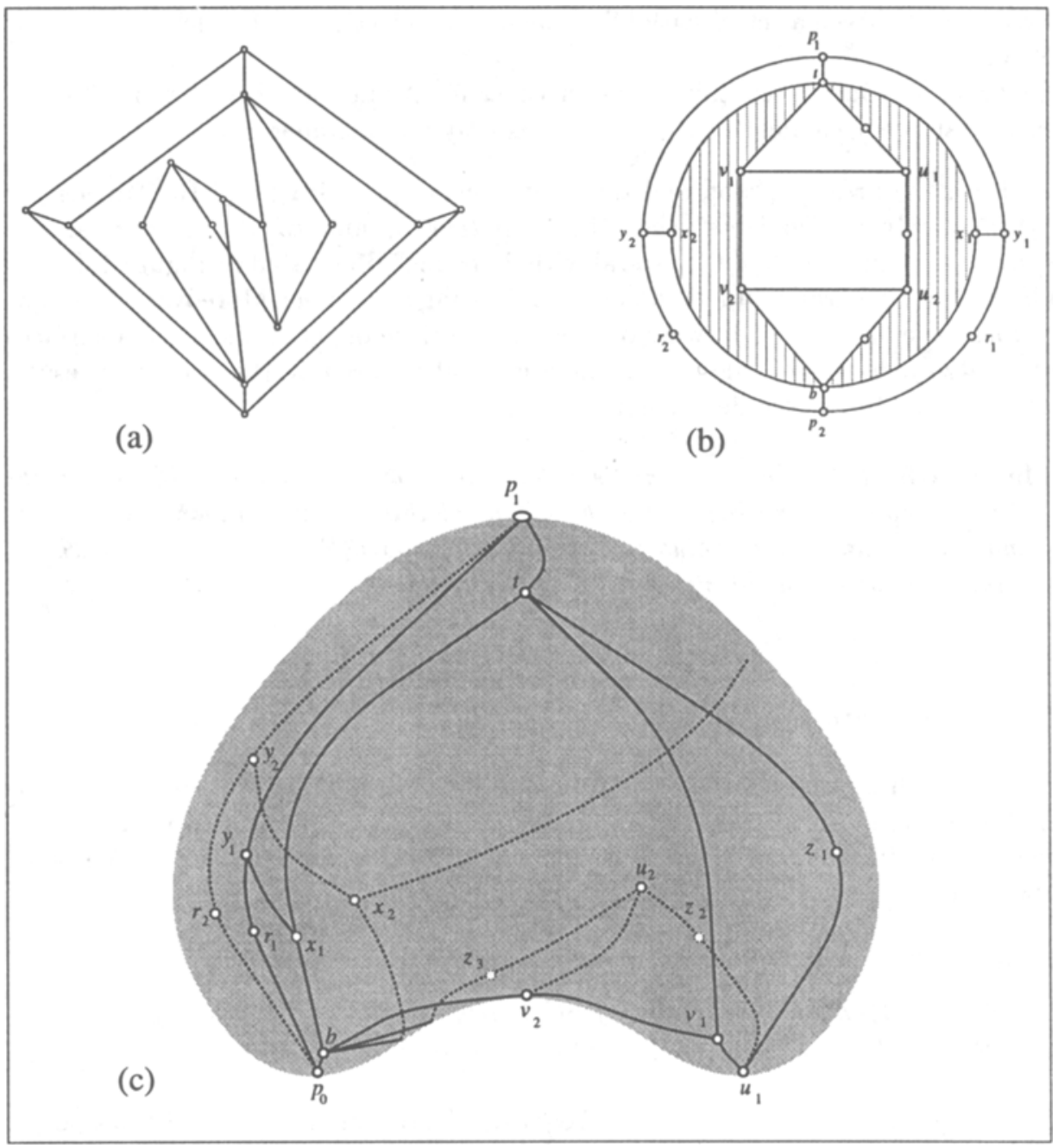

Fig. 4. (a) The Spiral $S(1,0)$. (b) The planar covering graph of $S(1,0)$ (c) An upward drawing of $S(1,0)$ on a surface with a saddle point.

Problem 6. Characterize those ordered sets which have an upward drawing on a sphere.

The bridge between the NP-completeness of upward planarity testing and upward sphericity testing is built over spirals. In effect, a spiral is spherical if and only if it is planar.

Problem 7. Characterize those ordered sets for which sphericity implies planarity. 


\section{Strategy of the Proof}

\section{Spherical Ordered Sets at a Glance}

What is the difference between the sphere $\left\{(x, y, z): x^{2}+y^{2}+z^{2}=1\right\}$ and an arbitrary homeomorph of it, that is, any smooth (compact and closed) surface in $\mathbf{R}^{3}$ of genus zero?

In a word, saddles.

An ordered set is spherical if it has an upward drawing on the unit sphere such that no two edges cross and all edges are monotonic paths with respect to the positive $z$-axis, the northerly direction (although the precise direction is arbitrary). [S. Foldes, I. Rival and J. Urrutia (1992)] showed that an ordered set is spherical if it has a top, a bottom, and its covering graph is planar. Actually, an ordered set is spherical if and only if, by adjoining new edges as needed, it can be extended to a directed acyclic graph, with top and bottom, and with planar covering graph. (This echoes a characterization of planar ordered sets in terms of planar lattices [D. Kelly (1987)] or, equivalently, st-graphs [G. di Battista and R. Tamassia (1988)].)

Fix an upward drawing (without crossing of its edges) of an ordered set $P$ on a surface $S$. To any covering edge $a \succ b$ in $P$ we associate two values, + to that end of the covering edge outgoing from $a$ and - to that end of it incoming to $b$. In this way, every covering edge acquires two values and, for every element $a \in P$, every incident covering edge associates, in this way, a sign (+ or - ), to a. A minimal element of $P$ will acquire all - values and a maximal element all + values. In general, to every element there is associated a circular sequence of +'s and -'s corresponding to a clockwise orientation in a neighbourhood about it. What is of importance is the number of alternations of +'s and -'s. Thus, we call an element ordinary just if this sequence consists of an interval of + 's and an interval of -'s - one alternation. An extremal element, that is, a maximal or a minimal element has no alternations at all. If an element's circular sequence has two or more alternations then it must be a saddle point - the surface on which the ordered set is drawn cannot be spherical.

On the boundary $v_{1}, v_{2}, \ldots, v_{m}$ of a face $F$ we consider all vertices which are extremal with respect to $F$, that is, all vertices $v_{i}$ such that either $v_{i-1} \succ v_{i}$ and $v_{i+1} \succ v_{i}$, or else, $v_{i-1} \prec v_{i}$ and $v_{i+1} \prec v_{i}$. Such a vertex is extremal if all of its neighbours are either larger or all are smaller. We call it interior with respect to this face $F$ if it has a neighbour $v$, not on the boundary of this face, with opposite sign, that is, $v \prec v_{i}$ if $v_{i-1} \succ v_{i}$ and $v_{i+1} \succ v_{i}$, while $v \succ v_{i}$ if $v_{i-1} \prec v_{i}$ and $v_{i+1} \prec v_{i}$.

An algorithmic analysis of a graph on a surface ultimately entails a triangulation of it. Any face which itself (as an ordered subset) contains a top and bottom can be triangulated without increasing the alternation about any of its vertices. The essential conclusion to which all of these observations lead is this.

An ordered set has an upward drawing on a sphere if and only if its covering graph has a triangulation with no saddle point at all. 


\section{Reduction Gadgets}

The $N P$-completeness reduction is based on standard techniques of "component design" (cf. [M. R. Garey and D. S. Johnson (1979)]).

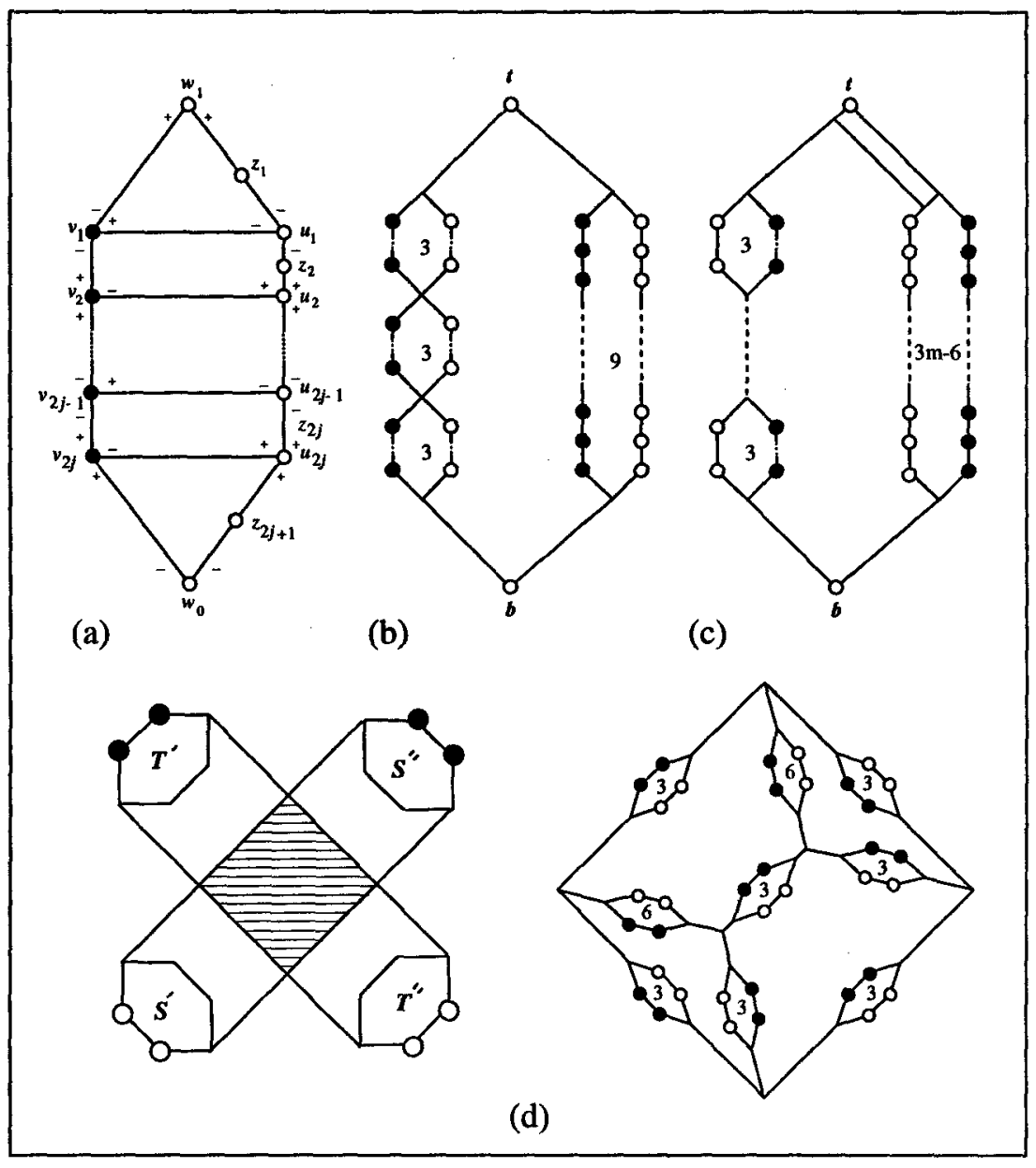

Fig. 5. (a) The spiral $S_{j}(b) Q(c) P(m)(d) R$

Here is the system of gadgets that are used in the reduction.

Loosely speaking, our aim is to construct a directed acyclic graph $P$ such that "flipping" its components corresponds to an instance of Exact Cover By 3-Sets.

- Spirals $S_{j}, j$ a positive integer, constitute the "flipping" components. 
- We collect four spirals at a time to build an ordered set (we call $Q$ ) to represent the elements of the set $X$. More precisely, $Q$ consists of three copies of $S_{3}$ and one $S_{9}$. (The point is that $Q$ has a spherical upward drawing if and only if either all of the three spiral components are "flipped" or none at all are "flipped".) For every member $c \in C$ we associate a copy of $Q$ and "flipping" the components shall correspond to choosing the member $c$ to be an element of the desired exact cover.

- Construct ordered sets $P(m)$, with $m+1$ spirals, $m$ of which are copies of $S_{3}$ and one $S_{3 m-6}$, intended to represent members of $C$, such that $P(m)$ has a spherical upward drawing if and only if exactly one of the spiral sets is "flipped". (For every element $x \in X$ there is a suitable copy of $P(m)$ and "flipping" a component corresponds to choosing the member of $C$ to cover the element $x$.

- We construct "communication" edges in $P$ to ensure that choices in the $Q$ components and the $P(m)$-components agree. Because these communication edges may cross, we replace each by a "crossover" $R$.

In summary, the directed acyclic graph is constructed in polynomial time and has a spherical upward drawing if and only if the corresponding instance of Exact Cover By 3-Sets has a positive solution.

\section{References}

K. A. Baker, P. C. Fishburn, and F. S. Roberts (1971) Partial orders of dimension 2, Networks (2), 11-28.

G. di Battista, W.-P. Liu and I. Rival (1990) Bipartite graphs, upward drawings, and planarity, Inform. Proc. Letters 36, 317-322.

G. di Battista and R. Tamassia (1988) Algorithms for plane representations of acyclic digraphs. Theoretical Computer Science 61, 175-198.

J. Czyzowicz. A. Pelc and I. Rival (1990) Planar ordered sets of width two, Math. Slovaca 40 (4), 375-388.

K. Ewacha, W. Li, and I. Rival (1991) Order, genus and diagram invariance, ORDER 8, 107-113.

S. Foldes, I. Rival and J. Urrutia, Light sources, obstructions, and spherical orders, Discrete Math. 102, 13-23.

M. R. Garey and D. S. Johnson (1979) Computers and Intractability : A Guide to the Theory of NP-completeness. Freeman,

A. Garg and R.Tamassia (1995) On the computational complexity of upward and rectilinear planarity testing, Lecture Notes in Computer Science (894) (eds. R. Tamassia and I. G. Tollis), pp. $286-297$.

A. Garg and R.Tamassia (1995) Upward planarity testing, ORDER (12(2)). 
S. Mehdi Hashemi and I. Rival (1994) Upward drawings to fit surfaces, in Orders, Algorithms, and Applications (ORDAL '94) (eds. V. Bouchitté and M. Morvan), Lecture Notes in Computer Science 831, Springer pp. 53-58.

J. Hopcroft and R. E. Tarjan (1974) Efficient planarity testing, J. Ass. Comp. Mach. 21 (4), 549-568.

M. D. Hutton and A. Lubiw (1991) Upward planar drawing of single source acyclic digraphs, Proc. 2nd A.C.M./ S.I.A.M. Symposium Discrete Appl. Math., pp. 203-211.

D. Kelly (1987) Fundamentals of planar ordered sets, Discrete Math. 63, 197216.

D. Kelly and I. Rival (1975) Planar lattices, Canad. J. Math. 27, 636-665.

A. Kisielewicz and I. Rival (1993) Every triangle-free planar graph has a planar upward drawing, ORDER 10, 1-16.

A. Lempel, S. Even and I. Cederbaum (1967) An algorithm for planarity testing of graphs, in Theory of Graphs, International Symposium, Rome (1966) (P. Rosenstiehl ed.), Gordon and Breach, pp. 215-232.

C. R. Platt (1976) Planar lattices and planar graphs, J. Comb. Th. Ser. B 21, 30-39.

K. Reuter and I. Rival (1991) Genus of orders and lattices, in Graph-Theoretic Concepts in Computer Science (R. Möhring ed.), Lect. Notes Comp. Sci. 484, pp. $260-275$.

I. Rival (1993) Reading, drawing, and order, in Algebras and Orders (I. G. Rosenberg ed.), Kluwer.

C. Thomassen (1989) Planar acyclic oriented graphs, ORDER 5, 349-361. 